\title{
A rare case of alveolar echinococcosis in a 14-year-old child
}

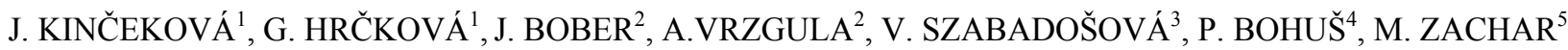

\author{
${ }^{1}$ Parasitological Institute, SAS, Hlinkova 3, 04001 Košice, Slovak Republic, E-mail: dr.kincek@zoznam.sk; ${ }^{2}$ First \\ Department of Surgery, Louis Pasteur University Hospital, SNP 1, 04190 Košice, Slovak Republic; ${ }^{3}$ Children Faculty \\ Hospital, Dept. of Infectious Diseases, SNP 1, 04001 Košice, Slovak Republic; ${ }^{4}$ Department of Pathology, Louis \\ Pasteur University Hospital, SNP 1, 04190 Košice, Slovak Republic; ${ }^{5}$ Dept. of Radiodiagnostic, Louis Pasteur \\ University Hospital, SNP 1, 04190 Košice, Slovak Republic
}

\begin{abstract}
Summary
We report the case of a 14-year-old girl who suffered from headache, fatigue, caught and a fever with body temperature up to $40{ }^{\circ} \mathrm{C}$ for a week. The patient had abdominal enlargement and clinical examination showed the hepatomegaly $+5 \mathrm{~cm}$. In ELISA assays employing $\mathrm{Em} 2+$ antigen, high titres of specific $\operatorname{IgG}$ antibodies to Echinococcus multilocularis somatic antigens were found in the serum. Western blot analysis also indicated on presence of alveolar echinococcosis (AE). CT examination showed expansive foci in the right liver lobe and histological examinations of the biopsy after PAS staining confirmed the presence of alveolar echinococcosis. After 320 days of chemotherapy with albendazole, CT revealed progression of parasitic foci and new abscess lesions inside parasitic cysts were detected, with some parts being necrotic. The surgical removing of foci was performed as following - the laparotomic revision, the removal of the right liver lobe with dilatation, reconstruction of $v$. cavae cum prothesis $\mathrm{PTFE}$ in length $12 \mathrm{~cm}$. Three months after the operation, the rest of the patient's left liver lobe had grownup and compensated at least a part of the removed liver.
\end{abstract}

Key words: alveolar echinococcosis; surgery; treatment; CT imaging; antibody response

\section{Introduction}

Alveolar echinococcosis is a life-threatening disease in humans caused by metacestode stage of tapeworm Echinococcus multilocularis. Lethality of natural AE reaches $94-100 \%$ within 10 - 15 years after diagnosis in untreated or inadequately treated human patients (Amman \& Eckert, 1996). In some endemic regions in Central Europe and Japan, the prevalence ranges from 2 to 40 cases per 100000 inhabitants (Eckert \& Deplazes, 1999). During 5 years $(1996$ - 2000), 10 new cases were reported in Austria, 48 in Germany and 16 in Poland (Kern et al.,
2003). The typical age of clinically confirmed patients for $\mathrm{AE}$ is between $50-70$ years, but infection is very rare in children. Studies and progress reports with regard to the surgical procedures are rare. Curative surgery is feasible only in the minority of patients, because frequently the parasitic lesion was already spread widely in the time of diagnosis. The other requirement for the surgical procedure is keeping the minimum distance of at least $2 \mathrm{~cm}$ between the lesion and the cut surface. Palliative surgery should therefore be reserved for the cases with complications that could not be managed by conservative and interventional treatment (Buttenschoen et al., 2001). In this study we report the case of 14-years-old child with very advanced stage of disease.

\section{Case report}

A 14-year-old female was born in Slovak Republic and lived in a village in the vicinity of a forest. In February 2006 she was admitted to the local hospital as she was suffering from headache, fatigue, caught and a fever with body temperature up to $40{ }^{0} \mathrm{C}$ for a week. The patient observed by herself the abdominal enlargement and clinical examination showed the hepatomegaly $+5 \mathrm{~cm}$. Based on ultrasonography examination of abdomen, the patient was re-hospitalised at an Oncological Clinic with diagnosis of a primary hepatic tumour. Examinations for the presence of several oncomarkers were negative.

CT examination revealed expansive foci large $12.5 \times 12.3$ $\mathrm{cm}$ localized in the right liver lobe with numerous calcifications inside the lesion (Fig.1). Computer tomography was performed using a CT scanner Somatom Sensation 10 (Siemens), rotation time of $0.5 \mathrm{~s}$ and multislice data acquisition with $\mathrm{UFC}^{(\mathrm{tm})}$ detector up to 10 slices simultaneously. The density of liver foci and its extent following $\mathrm{CT}$ examination (40 HU) indicated on possible parasitic origin. To detect Echinococcus specific 


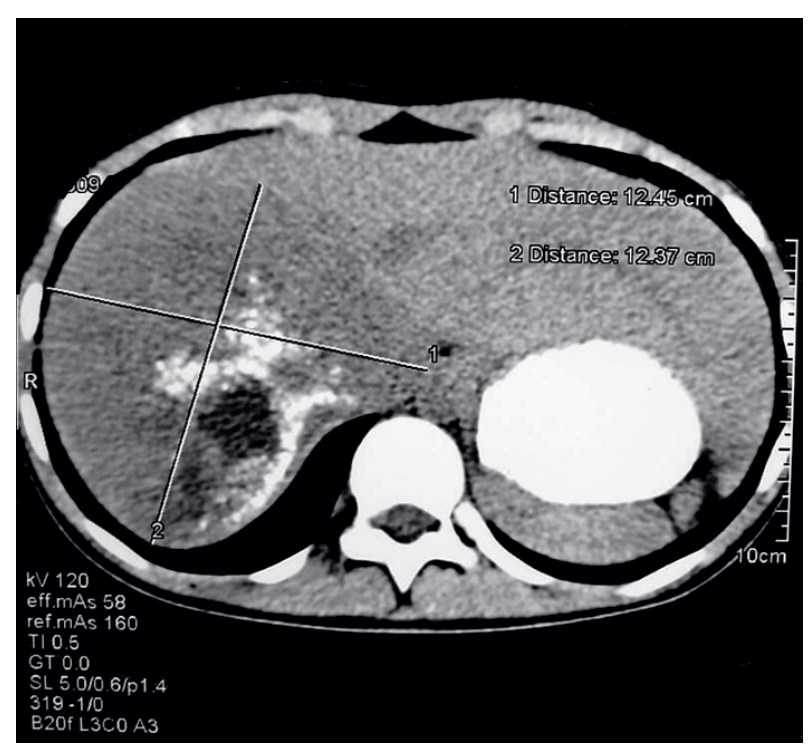

Fig.1. Abdominal CT scan - expansive foci in the right liver lobe 12.5 $\mathrm{x} 12.3 \mathrm{~cm}$ in size. Numerous calcifications were seen in the lesion. Cystoidal lesions with lower density in average of $3 \mathrm{~cm}$ indicate the necrotic character

antibodies, an Em2+ ELISA Kit (Bordier Affinity Products, Crissier, Switzerland) was performed in accordance with the manufacturer's instructions. Em2+ ELISA detected high titers of antiparasitic IgG antibodies in serum during the whole course of chemotherapy. The specificity of serum antibodies was examined using the commercial Echinococcus Western blot IgG Kit (LDBIO Diagnostics, Lyon, France). IgG serum antibodies from patient cross-reacted with parasitic antigens of molecular weight (MW) $26-28 \mathrm{kDa}, 16$ and $18 \mathrm{kDa}$, and such immuneblot pattern indicates the presence of E. multilocularis infection.

In March 2006, the patient was subjected to the laparotomic revision of abdomen, which showed the

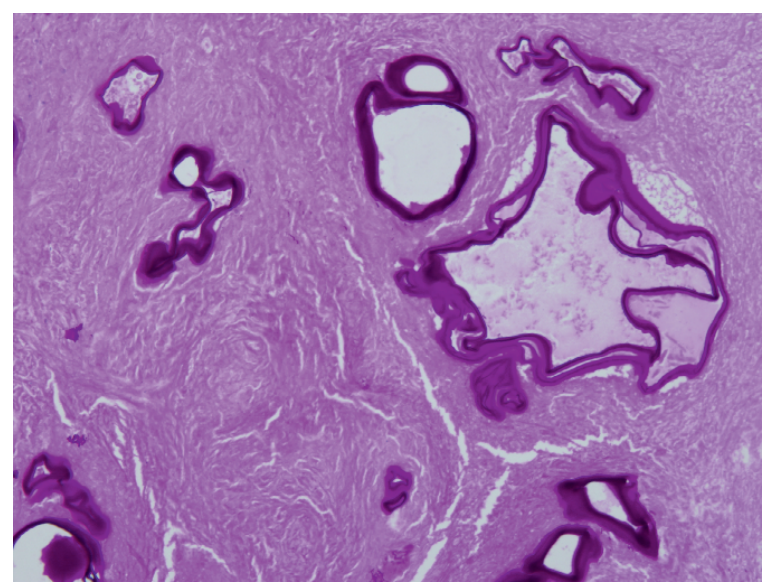

Fig. 2. Histological examination. Lamellar membranes stained with period acid-Schiff reagent with diastase (PAS-D), original magnification x 100. Alveolus wall showed outer lamellar acellular membrane, which was not stained with PAS-D. Thin inner cellular germinal layer was only focally preserved. No protoscoleces were found presence of solid, tumour-like infiltration in the right and partially in the left liver lobes. Wedge excision of liver tissue $16 \times 11 \times 8 \mathrm{~mm}$ in size, which had a cartridge-like and yellowish appearance, nodular on cut, was submitted to pathological examination. Sections from formalin-fixed and paraffin embedded tissue were stained with haematoxylin/eosin or periodic acid-Schiff reagent with diastase (PAS - D staining). Microscopically many alveoli of different sizes and shapes were found (Fig. 2) but no protoscoleces of E. multilocularis were detected. Chemotherapy with albendazole (Zentel, 600mg/day in two doses) was initiated and after one year of discontinuous administration of the drug, progression of the parasitic lesion occurred. CT examination (Fig. 3) showed enlarged hypodense-cystiform part of the lesion, indicating on its coliquation.

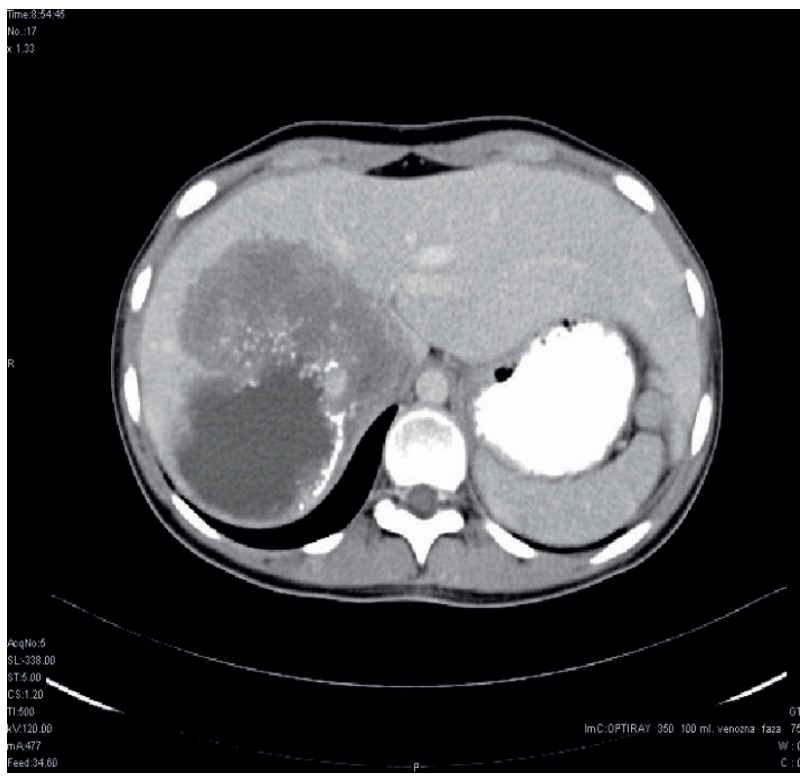

Fig.3. Abdominal CT scan in the venous phase of contrast examination. Hypodense parts show places with lower density indicating the necrotic character

In May 2007, the patient was admitted for surgical treatment of the liver cyst (Fig. 4a, b) and the massive right hemi-hepatectomy was performed followed by the restorative operation on vena cava inferior due to the Echinococcus infiltrations, and replacement of ventral wall $12 \mathrm{~cm}$ in size. The vital functions of patient were stabilized and the extensive amount of ascitic fluid from the abdomen was drained. To avoid the risk of thrombosis in the vena cava inferior, high doses of heparine (with low molecular weight) were administered. However on day 15 after operation, the patient conditions were aggravated due to bleeding into abdominal cavity. The reason of this, found during revision, was a small hole in arteria hepatica $l$. sin. In further follow-up, the amount of ascitic drainage diminished without presence of bleeding and normalisation of biochemical parameters occurred within 33 days of hospitalisation after surgical treatment of AE. 

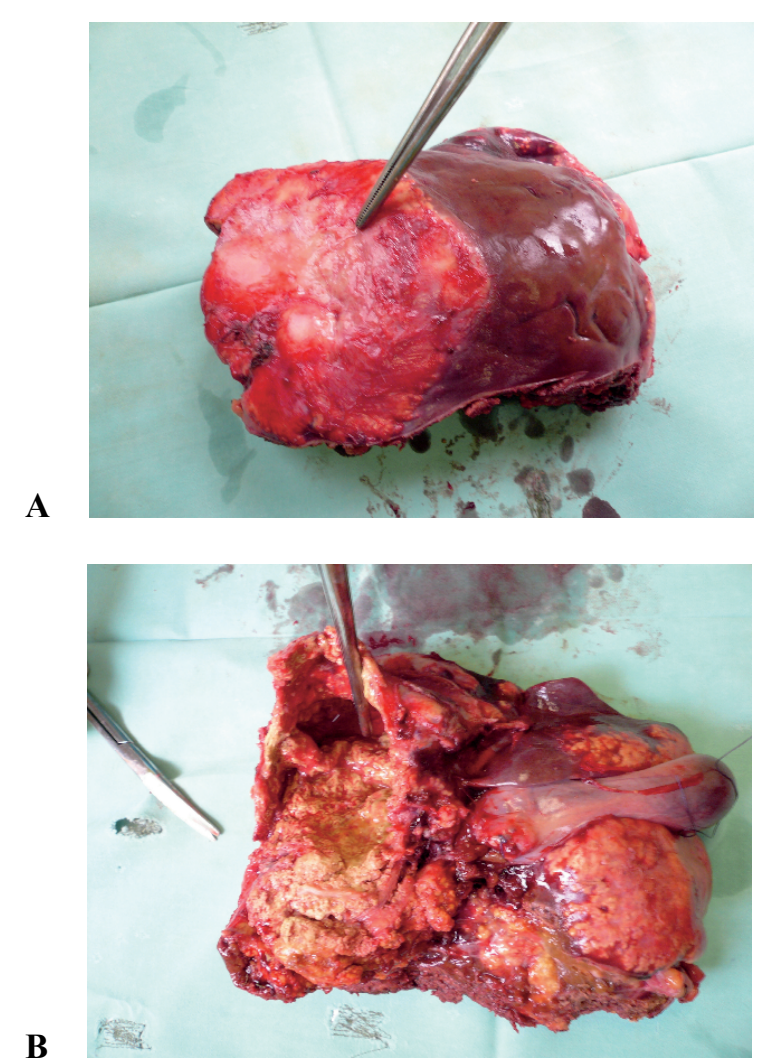

Fig. 4. Pathological findings. A - Section of liver tissue with alveolar echinococcosis. Macroscopic appearance of multiple small and larger cysts. B - Central disruption of the tissue and colliquation of lesion $(16 \times 14 \mathrm{~cm})$

The control CT examination performed in August 2007, i.e. 3 months after surgery (Fig. 5), showed that remnant of left liver lobe expanded and compensated for the removed right lobe. On appearance it had normal structure of arterial system without dilatation of biliary ducts, and also without presence of any lesions indicating AE. Moreover, also serological examination for presence of specific IgG antibodies was negative.

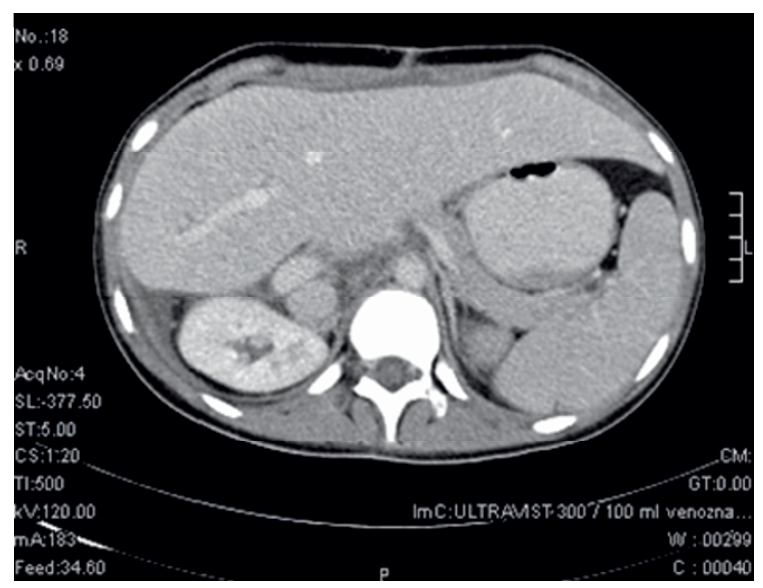

Fig.5. Abdominal CT scan. Remnant of the left liver lobe has grown and compensated a part of removed right lobe. It has normal structure of arterial system without dilatation of biliary ducts, and also without presence of any AE lesions

\section{Discussion}

Echinococcus multilocularis infiltrates gradually into the liver tissue in much the same way as a malignant tumour. The neighbouring organs outside the liver, such as the porta hepatis, diaphragm, pancreas or vena cava inferior can also be involved. Most common sites for blood borne metastases are lungs and brain but lymphogenous metastasis of the porta hepatis lymph node, peritoneal cavity and mesentery can be found (Jiang \& Jones, 2005). Alveolar echinococcosis is a rather uncommon parasitic disease in the Slovak Republic. The first human cases of AE were recorded in Slovak Republic in 2000 and within the following period we have diagnosed 9 new cases of all age categories (Kinčeková et al., 2006).

Of these 9 patients were elderly or in middle age, and only one case was 14 year old child. In spite of her young age, the enormous size of parasitic lesion indicated that she was infected in her early childhood as she lives close to a forest, inhabited by red foxes. We suppose that source of infection was fruit or vegetables contaminated by parasitic eggs. In case of AE, clinical symptoms of the disease are rarely manifested in the early stage of infection, which could last sometimes 10-15 years. However, in a majority of cases the disease is diagnosed in elderly patients and only rarely in children (Bresson-Hadni et al., 2000). Sailer et al., (1997) described AE case in a six-year-old-girl in Austria and diagonosis was confirmed histologically only. Child suffered from acquired immunodeficiency syndrome, which probably contributed to ecinococcusnegative serological tests. The patient described in this study had no immunodeficiency syndrome and responded to the infection by very high titres of specific antibodies.

Diagnosis of AE is mainly based on the identification of the parasitic lesions using imaging techniques (CT, US) and on detection of the specific antibodies (Gottstein, 1992). The therapy is quite complicated. Albendazole and mebendazole are drugs of choice, recommended for the treatment of both cystic (CE) and alveolar echinococcosis (AE), whereas in the case of AE, these drugs have a primarily parasitostatic rather than parasitocidal effect (Nakajima et al., 1993). The disease is curable only if AE is diagnosed in an early stage and/or the parasitic lesions are completely surgically removed (Eckert et al., 2001). In the case of our patient, the parasitic cyst was resistant to albendazole treatment and further expansion inside cyst was recorded on CT. A thick lamellar layer enclosing parasitic tissue and adjacent host-derived fibrous layer probably inhibited penetration of albendazole into the cyst. The failure of chemotherapy and the expansive character of the disease indicated surgical removal of infected liver tissue. This was successful although nearly $70 \%$ of liver was removed. The liver is an organ with an enormous regenerating capacity and after experimental hepatectomy of one liver lobe, the new liver parenchyma are formed within a few weeks (Buttenschoen et al., 2001). Indeed, 3 months after operation, CT examination showed that rest of the left liver lobe of our patient has grown and 
compensated for at least part of removed liver. Young age and absence of other diseases probably contributed to such rapid liver regeneration. Even in such cases, WHO recommended pre-and postoperative chemotherapy at least for 2 years (WHO, 1996). Radical treatment approach proved to be successful in this case, as neither new foci, nor rise of specific antibodies was observed so far. Patient is under continuous medical and serological control.

\section{Acknowledgement}

The work was supported by the Science Grant Agency VEGA 2/7186/27 and the Slovak Research and Development Agency under the contract No. APVV-51027605 .

\section{References}

AMMAN, R.W., ECKERT, J. (1996): Echinococcus. In WeINSTOCK, J.V. (Ed): Parasitic diseases of the liver and intestines. Saunders, Philadelphia: 655 - 689

Bresson - HAdni, S., Vuitton, D. A., BARtholomot, B., Godart, D., Meyer, J. P., Hrusovsky, S., Becker, M. C., Miguet, J. P. (2000): A 20-year history of alveolar echinococcosis in humans. Analysis of a series of 117 patients from eastern France. Eur. J. Gastroenterol. Hepatol., 12: $327-336$

Buttenschoen, K., Schorcht, P., Reuter, S., Buttenschoen, Carli, D., Kern, P., Beger, H. G. (2001): Die chirurgische Therapie der alveolären Echinokokkose und deren Langzeitergebnisse. Chirurg, 72: $566-572$

ECKERT, J., DePlazes, P. (1999): Alveolar echinococcosis in humans: the current situation in Central Europe and the need for countermeasures. Parasitol. Today, 15: 315 - 319 Eckert, J., GotTstein, B., HeAth, D., LiU, F. J. (2001): Prevention of echinococcosis in humans and safety precations. In: ECKERT, J., GEMMEL, M. A., MESLIN, F. X., PAWLOWSKI, Z. S. (Eds): WHO/OIE Manual on Echinococcosis in Humans and Animals: A Public Health Problem of Global Concern. WHO and OIE, Paris: $238-247$

GotTSTEIN, B. (1992): Echinococcus multilocularis infection: immunology and immunodiagnosis. Adv. Parasitol., 31: $321-380$

JiAnG, C. P., Don, M., JONES, M. (2005): Liver alveolar echinococcosis in China: Clinical aspect with relative basic research. World J. Gastroenterol., 11: 4611 - 4617

Kern, P., BARdonnet, K., Renner, E., Auer, H., Pawlowski, Z., Amman, R. W., Vuiton, D. A., Kern. P. (2003): European Echinococcosis Registry: human Alveolar Echinococcosis, Europe, 1982-2000. Emer. Inf. Dis., 9: $343-349$

KinčEKovÁ, J., HrČKOVÁ, G., Auer, H., Szilágyiová, M., HudačKová, J., Stanislayová, M., PoláčEK, H., ŠIMEKOVÁ, K. (2006): Clinical and serological study of human alveolar echinococcosis in Slovakia in relation to the outcome of chemotherapy. Helminthologia, 42: $213-$ 219

NAKAJima, Y., SATO, N., UChinO, J. (1993): Stage of the disease. In UCHINO, J., SATO, N. (Eds): Alveolar echinococcosis of the liver. Hokkaido University Medical Library Series, Sapporo: 115 - 119

World HeAlth ORGANisAtion (1996): Guidelines for treatment of cystic and alveolar echinococcosis in humans. Bull. World Health Organ., 74: 231 - 242 